Article

\title{
The Role of Magnetic Islands in Collisionless Driven Reconnection: A Kinetic Approach to Multi-Scale Phenomena
}

\author{
Ritoku Horiuchi \\ National Institute for Fusion Science, 322-6 Oroshi, Toki 509-5292, Japan; horiuchi.ritoku@toki-fs.jp; \\ Tel.: +81-572-58-235
}

Received: 13 February 2018; Accepted: 17 April 2018; Published: 21 April 2018

\begin{abstract}
The role of magnetic islands in collisionless driven reconnection has been investigated from the standpoint of a kinetic approach to multi-scale phenomena by means of two-dimensional particle-in-cell (PIC) simulation. There are two different types of the solutions in the evolution of the reconnection system. One is a steady solution in which the system relaxes into a steady state, and no island is generated (the no-island case). The other is an intermittent solution in which the system does not reach a steady state, and magnetic islands are frequently generated in the current sheet (the multi-island case). It is found that the electromagnetic energy is more effectively transferred to the particle energy in the multi-island case compared with the no-island case. The transferred energy is stored inside the magnetic island in the form of the thermal energy through compressional heating, and is carried away together with the magnetic island from the reconnection region. These results suggest that the formation of a magnetic island chain may have a potential to bridge the energy gap between macroscopic and microscopic physics by widening the dissipation region and strengthening the energy dissipation rate.
\end{abstract}

Keywords: magnetic reconnection; magnetic island; multi-scale physics; open system; energy transfer

\section{Introduction}

A physical system, in which magnetic reconnection takes place as one of key processes, evolves dynamically with time and reveals various interesting features such as rapid energy release, intermittency, self-organization, and so on [1,2]. For triggering magnetic reconnection, microscopic physics are required to generate an electric resistivity in the vicinity of a reconnection point. On the other hand, once magnetic reconnection occurs, the field topology changes on a macroscopic scale and global plasma transport takes place. Furthermore, the macroscopic plasma dynamics, in turn, affect the microscopic reconnection physics through the compression of the reconnection region, the energy inflow into and/or outflow from the reconnection region [3,4], turbulence and plasma instabilities [5,6], and so on.

Thus, magnetic reconnection itself is a typical multi-scale phenomenon including microscopic electron dynamics through global plasma transport. One of the big issues in magnetic reconnection studies is how to construct the self-consistent model for these multi-scale processes, because there are large scale gaps in space and time between the microscopic and the macroscopic processes. For example, the dissipation time due to the binary collision in the solar corona is of the order of 1 million years. On the other hand, a solar flare, which is expected to be controlled by magnetic reconnection, takes place in a time frame ranging from a few minutes to a few hours [7]. Sweet [8] and Parker [9] separately proposed the first quantitative model of magnetic reconnection in two-dimensional geometry to solve this scale gap problem. After them, many researchers tried to construct various models to solve this 
problem [2], but the multi-scale physics of magnetic reconnection are not fully understood and remain an unsolved issue [10].

The energetics of magnetic reconnection phenomena in nature are a typical example. The microscopic dissipation region, in which the electromagnetic energy is effectively transferred to the plasma energy, is localized in the vicinity of the reconnection point and its spatial scale ranges from one electron Larmor radius to a few ion Larmor radii at most. Therefore, the total amount of the released energy is too small to explain the wide-ranging energy spectrum of magnetic reconnection phenomena such as solar flares [7].

Because the microscopic kinetic regions are surrounded by the global macroscopic system, the microscopic dissipation process itself is always under the influence of macroscopic physics. It is easy to expect that particle acceleration and heating may be enhanced through their mutual interaction, because it may generate many reconnection points and widen the dissipation region through the formation of a magnetic island chain and plasma instabilities in some cases [6,11-17]. Thus, the hierarchically connecting chains of magnetic islands represent an attractive idea to explain the wide-ranging energy spectrum $[11,12,18]$. The problems are determining how and under what conditions magnetic are islands generated and the energy dissipation enhanced.

In order to solve these problems, we developed a particle-in-cell (PIC) simulation model for the magnetic reconnection study in an open system, known as "PASMO", in which the information of the macroscopic physics is introduced for boundary conditions, and only a microscopic reconnection system is solved under given boundary conditions $[3,4,19]$. This PASMO code enables us to investigate the long time scale behavior of magnetic reconnection as well as the microscopic triggering mechanism of magnetic reconnection.

In this paper we investigate the role of magnetic islands in collisionless driven reconnection from the standpoint of a kinetic approach to multi-scale phenomena using the PASMO code, focusing in particular on energy transfer.

\section{Simulation Model}

Our PIC simulation model relies on the explicit electromagnetic scheme [20] and is implemented on two-dimensional Cartesian coordinates $(x, y)$. As an initial condition we assume one-dimensional Harris-type equilibrium [21] with an anti-parallel magnetic field along the $x$-axis and a uniform guide field along the $z$-axis as

$$
\begin{gathered}
P(y)=P_{0}+\frac{B_{0}^{2}}{8 \pi} \operatorname{sech}^{2}(y / L), \\
\mathbf{B}(y)=\left(B_{0} \tanh (y / L), 0, B_{z 0}\right),
\end{gathered}
$$

where $B_{0}, B_{z 0}$, and $P_{0}$ are constant, and $L$ is the scale height along the $y$-axis. The initial particle distribution is assumed to be a shifted Maxwellian with spatially constant temperature $\left(T_{i 0}=T_{e 0}\right)$ and an average particle velocity which is equal to the diamagnetic drift velocity. In the PASMO code, two types of the open boundary conditions are adopted, i.e., the inflow boundary condition at the upstream boundary $\left(y= \pm y_{b}\right)$, and the Neumann-type (floating) condition at the downstream boundary $\left(x= \pm x_{b}\right)[4,22]$. An external driving field $E_{d}(x, t)$ is assumed at the upstream boundary in order to supply plasmas into the system, which is controlled by three parameters as, i.e., (1) an initial non-uniformity scale of the driving field, which is called the inflow window size $\left(X_{\text {slot }}\right),(2)$ expansion speed of the driving field along the $x$-axis $\left(v_{\text {exp }}\right)$, and (3) a constant inflow rate of the magnetic flux $\left(E_{d 0}\right)$.

In order to investigate the role of magnetic islands, we have carried out four simulation runs by changing the inflow window size and the guide magnetic field, using the PASMO code. The typical simulation parameters for the four cases are listed in Table 1, where $\rho_{i 0}$ is the ion Larmor radius associated with the total magnetic field $B_{t}\left(=\sqrt{B_{0}^{2}+B_{z 0}^{2}}\right), l_{m i}\left(=\sqrt{L \rho_{i 0}}\right)$ is the ion meandering orbit amplitude [3], the spatial scales are normalized by $c / \omega_{c e 0}$, and $\omega_{c e 0}$ is the electron cyclotron frequency 
associated with $B_{0}$. The ratio $X_{\text {slot }} /\left(2 l_{m i}\right)$ is introduced as a parameter to control the aspect ratio of the current sheet.

Table 1. Simulation parameters, where $\rho_{i 0}$ is the ion Larmor radius associated with the total magnetic field $B_{t}\left(=\sqrt{B_{0}^{2}+B_{z 0}^{2}}\right), l_{m i}\left(=\sqrt{L \rho_{i 0}}\right)$ is the ion meandering orbit amplitude [3], the spatial scales are normalized by $c / \omega_{c e 0}$, and $\omega_{c e 0}$ is the electron cyclotron frequency associated with $B_{0}$. The last column stands for the status of magnetic island formation.

\begin{tabular}{ccccccc}
\hline Case & $\boldsymbol{B}_{\boldsymbol{z} \mathbf{0}} / \boldsymbol{B}_{\mathbf{0}}$ & $\boldsymbol{L}$ & $\boldsymbol{\rho}_{\boldsymbol{i 0}}$ & $\boldsymbol{X}_{\text {slot }}$ & $\boldsymbol{X}_{\text {slot }} /\left(2 \boldsymbol{l}_{\boldsymbol{m i}}\right)$ & Island \\
\hline A & 0.5 & 1.098 & 0.9245 & 8.786 & 4.360 & no island \\
B & 2.0 & 1.098 & 0.4623 & 8.786 & 6.166 & no island \\
C & 0.5 & 1.098 & 0.9245 & 17.57 & 8.720 & multi-island \\
D & 2.0 & 1.098 & 0.4623 & 17.57 & 12.33 & multi-island \\
\hline
\end{tabular}

According to the previous simulations [4,22], collisionless reconnection is found to be triggered when the current sheet is compressed by an external driving source and approaches a kinetic regime with a typical spatial scale comparable to the ion Larmor radius. The value of the scale height is determined so as to reproduce the same situation as the previous simulation runs. Simulation studies of collisionless driven reconnection with no guide magnetic field $[3,4]$ have also revealed that a steady reconnection is realized for small inflow window sizes of $X_{\text {slot }} /\left(2 l_{m i}\right)=5.19$ and 7.79 , while the reconnection system becomes intermittent for a large inflow window size of $X_{\text {slot }} /\left(2 l_{m i}\right)=10.39$. In order to examine whether this condition holds for a finite guide magnetic field, the values of the parameters $X_{\text {slot }}$ and $B_{z 0} / B_{0}$ are chosen. The other important parameters are fixed as: the mass ratio $M_{i} / M_{e}=100, \omega_{p e} / \omega_{c e 0}=6, v_{\exp }=v_{A x}$, and $E_{d 0}=-0.04 B_{0}$, where $\omega_{p e}$ is the electron plasma frequency associated with the initial number density at the center of the current sheet, and $v_{A x}$ is the initial Alfvèn velocity associated with $B_{0}$ at the upstream boundary. The condition $v_{\exp }=v_{A x}$ is determined from the assumption that the frozen-in condition is satisfied and the plasma beta is much smaller than a value of 1 near the upstream boundary. The simulation domain size is $X_{l} \times Y_{l}=26.357 c / \omega_{c e 0} \times 4.393 c / \omega_{c e 0}$. The total number of particles, which is a function of time in the PASMO code, is 18 million in the initial profile and can increase to up to 90 million at a maximum, including the uniform background particles of 20 per each space cell.

In the PASMO code, the space, time, velocity, and electromagnetic fields are normalized by $R_{N}=c / \omega_{c e 0}, T_{N}=1 / \omega_{c e 0}$, the light velocity $c$, and $B_{0}$, respectively. Thus, the current density and the energy density in the two-dimensional system are expressed in the normalization unit of $q c / R_{N}^{2}$ and $M_{e} c^{2} / R_{N}^{2}$, respectively, where $q$ is the electron charge. Because a few simulation runs are carried out for the different guide magnetic fields in this study, the unit of time is changed to $1 / \omega_{p e}$ from $1 / \omega_{c e 0}$ in this paper.

The time evolution of the external driving field $E_{d}(x, t)$ for Case B is plotted in Figure 1 . The driving field is set to zero initially, and grows while keeping a bell-shaped spatial structure in some time interval $\left(0<\omega_{p e} t<2584\right)$, reaching a constant and uniform profile of $E_{d}(x, t)=E_{d 0}=\left(-0.04 B_{0}\right)$. 


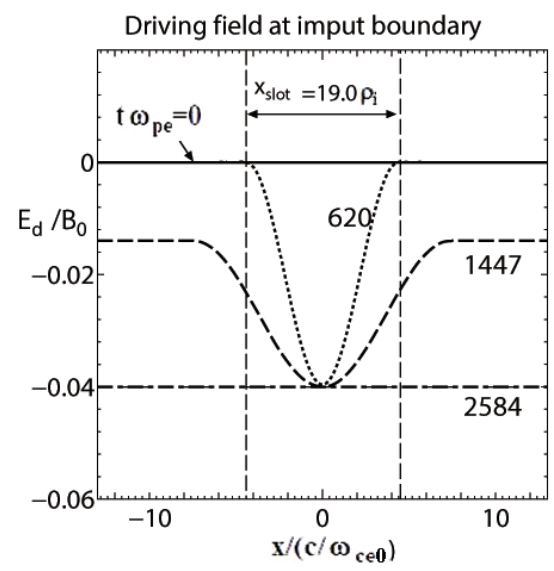

Figure 1. Time evolution of the external driving field $E_{d}(x, t)$ imposed at the upstream boundary for Case B, where $X_{\text {slot }}=19.0 \rho_{i 0}, v_{\exp }=v_{A x}$, and $E_{d 0}=-0.04 B_{0}$.

\section{Simulation Results}

As listed in Table 1 , when the ratio $X_{\text {slot }} /\left(2 l_{m i}\right)$ becomes larger than the critical value $R_{c r}$ which is between 6.166 and 8.720, an intermittent behavior accompanied by the multi-island formation appears in the dynamical evolution of a reconnection system. This result is consistent with the previous simulation results [4] and suggests that the tearing instability may play an important role in the formation of the multi-island state $[6,18,23]$. In this section we examine the role of magnetic islands in collisionless driven reconnection by comparing the multi-island case with the no-island case. Since the results of Cases $B$ and $D$ are similar to those of Cases $A$ and $C$, respectively, we focus on the simulation results in Cases $\mathrm{B}$ and $\mathrm{D}$ with $B_{z 0} / B_{0}=2.0$ hereafter.

\subsection{Two Types of Solutions}

Figure 2 plots the temporal evolutions of the reconnection electric field $E_{z}$ (dotted) and the electron current density $J_{e z}$ (solid) at the reconnection point for Cases B (left) and D (right), where the reconnection electric field is defined by the off-plane component of the electric field at the reconnection point. After the initial transient phase, the reconnection electric field reaches a constant value for the Case B, which is equal to the driving electric field imposed at the upstream boundary. This result means that the flux inflow rate is balanced with the reconnection rate and thus, the system relaxes into a steady state. On the other hand, the reconnection electric field fluctuates around the driving electric field with a large amplitude, and never reaches a constant value for Case D.
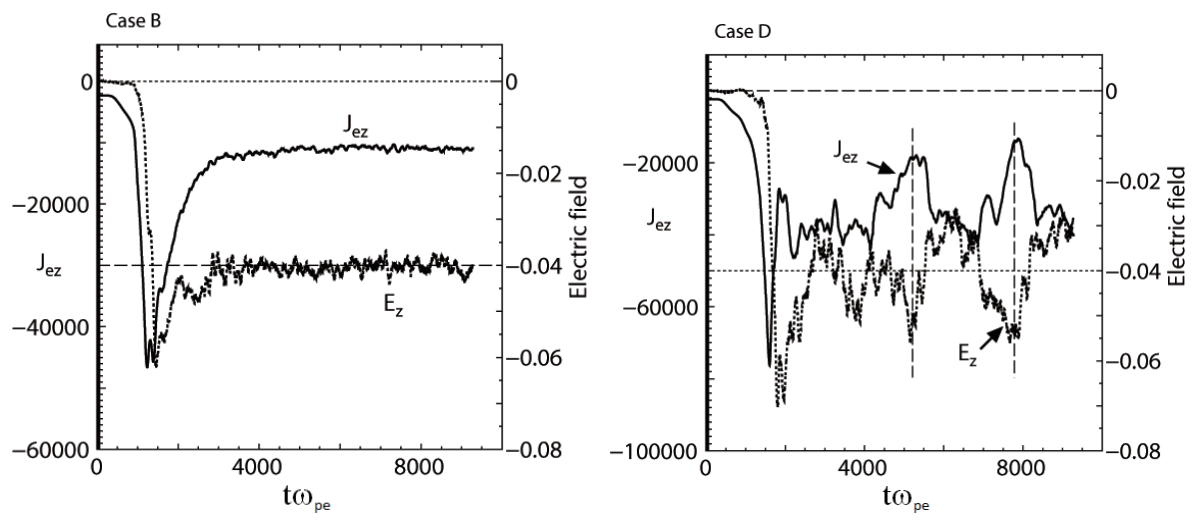

Figure 2. Temporal evolutions of the reconnection electric field $E_{z}$ (dotted) and the electron current density $J_{e z}$ (solid) at the reconnection point for Cases B (left) and D (right). The vertical dashed lines in the right panel correspond to the period when the size of a magnetic island reaches its local maximum. 
The vertical dashed lines in the right panel of Figure 2 indicate the periods when the reconnection rate reaches its local maximum value and the absolute value of the current density becomes locally minimum. Let us examine what happens at these periods in the simulation domain using the data at $\omega_{p e} t=7753$. The spatial profiles of the electron current density (color contours) and the magnetic field lines (black lines) at $\omega_{p e} t=7753$ are drawn in Figure 3, where the top and bottom panels correspond to the profiles for Cases B and D, respectively. It is clearly seen in Figure 3 that a large magnetic island is formed around this period in the Case D, while there is no magnetic island in the Case B. Thus, a large magnetic island is generated twice during the simulation run for the Case D. It is worth noting that the effective resistivity at the reconnection point, $E_{z} / J_{e z}$, reaches a maximum when the magnetic island is generated. In this way, there are two different types of the solutions in the evolution of the reconnection system. One is a steady solution in which the system relaxes into a steady state, and no island is generated. This solution corresponds to Cases A and B with the small inflow window size of $X_{\text {slot }} /\left(2 l_{m i}\right)<R_{c r}$. The other is an intermittent solution in which the system does not reach a steady state, and magnetic islands are frequently generated in the central region of the current sheet. This solution is realized in Cases C and D with the large inflow window size of $X_{\text {slot }} /\left(2 l_{m i}\right)>R_{c r}$.
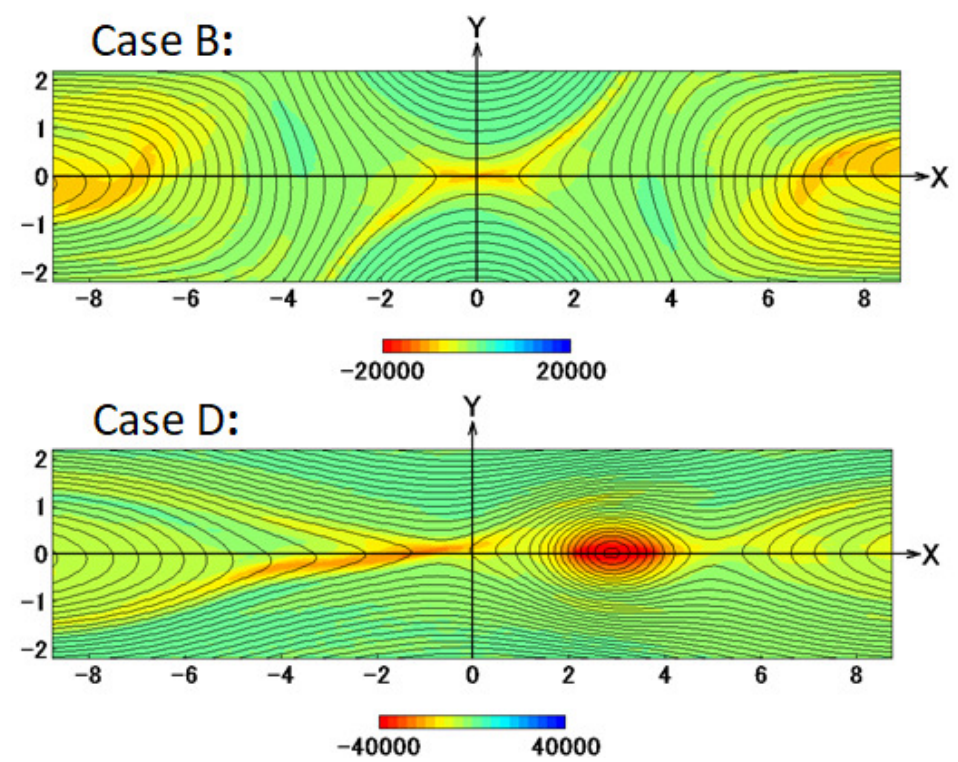

Figure 3. Spatial profiles of the electron current density (color contours) and the magnetic field lines (black lines) at $\omega_{p e} t=7753$ for Cases B (top) and D (bottom).

\subsection{Role of Magnetic Islands}

Let us examine the role of the magnetic island in the energy transfer process controlled by magnetic reconnection. The total energy transferred from the electromagnetic field energy to the particle energy during the time interval between $t-\Delta t$ and $t, d W_{s}(t)$, is given by

$$
\begin{aligned}
d W_{s}(t) & =\int_{t-\Delta t}^{t} d t \int_{S_{d}} d x d y\left\{e_{s} n_{s} \mathbf{E} \cdot \mathbf{u}_{s}\right\} \\
& =W_{s}(t)-W_{s}(t-\Delta t)+\int_{t-\Delta t}^{t} d t \int\left\{\mathbf{F}_{S}(t, \mathbf{x}) \cdot d \mathbf{s}\right\},
\end{aligned}
$$

where the subscript $s(=$ ele/ion $)$ stands for the electrons or the ions, and $e_{s}, n_{s}, \mathbf{u}$, and $d \mathbf{s}$ are the charge, the number density, the fluid velocity of the species $s$, and the line element surrounding the 
integral domain $S_{d}$, respectively. Here, the particle energy $W_{s}$ and the particle energy flux $\mathbf{F}_{s}$ of the species $s$ are defined as

$$
\begin{gathered}
W_{s}(t)=\int_{S_{d}}\left\{\frac{1}{2} \rho_{m s} \mathbf{u}_{s}^{2}+\varepsilon_{s}\right\} d x d y, \\
\mathbf{F}_{s}(t, \mathbf{x})=\left\{\frac{1}{2} \rho_{m s} \mathbf{u}_{s}^{2}+\varepsilon_{s}\right\} \mathbf{u}_{s}-\sigma_{s} \cdot \mathbf{u}_{s}+\mathbf{q}_{s},
\end{gathered}
$$

where $\rho_{m}, \varepsilon, \sigma_{i, j}$, and $\mathbf{q}$ are the mass density, the thermal energy density, the stress tensor, and the heat flux, respectively. The total energy transferred from the electromagnetic field energy to the electrons $\left(d W_{\text {ele }}\right.$, dotted) and ions $\left(d W_{i o n}\right.$, solid) are plotted as a function of the time for Cases B (left) and D (right) in Figure 4, where the time interval used for the energy integral is $\Delta t=10.34 \omega_{p e}^{-1}$, and the integration is taken over the square domain $S_{d}$ defined by $-8.80<x /\left(c / \omega_{c e 0}\right)<8.80$ and $-2.12<y /\left(c / \omega_{c e 0}\right)<2.12$, which is located inside the simulation domain and the central current sheet is also included. It is clear in Figure 4 that the magnetic energy is more effectively transferred to the particle energy in the multi-island case (Case D) compared with that in the no-island case (Case B).
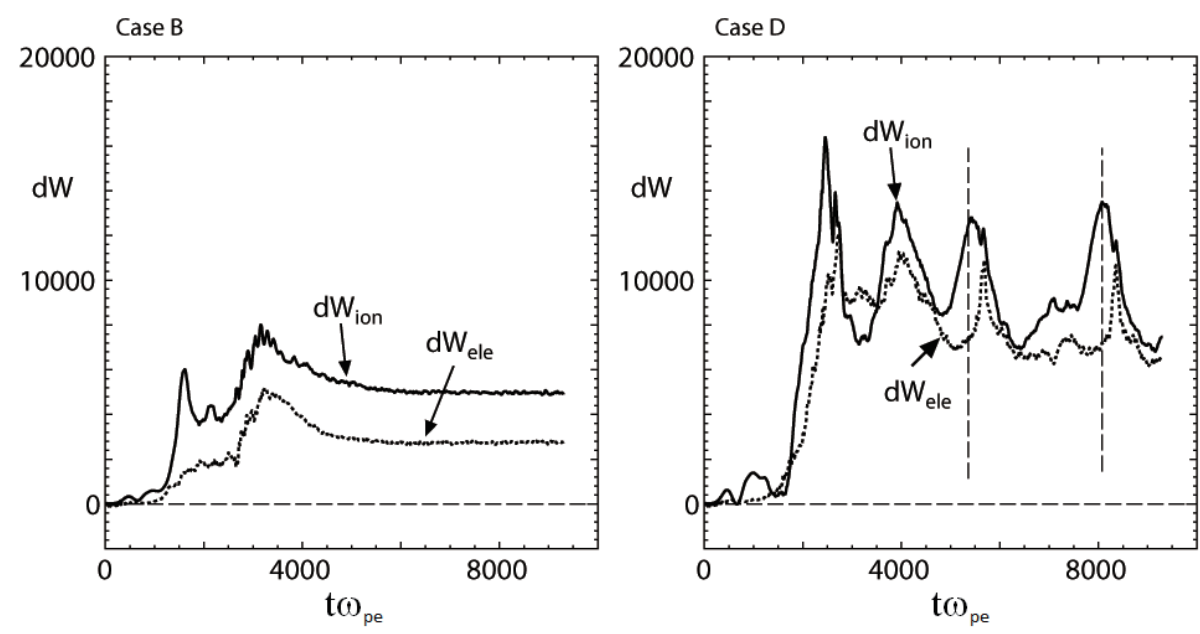

Figure 4. Temporal evolution of the total energy transferred from the electromagnetic energy to the electrons (dotted) and ions (solid) during the time interval between $t-\Delta t$ and $t$ for Cases B (left) and $\mathrm{D}$ (right) with $\Delta t=10.34 \omega_{p e}^{-1}$.

Equations (3) and (4) indicate that the total energy transferred from the electromagnetic field to the particles consists of (1) the energy carried away from the square domain $S_{d}$ in the form of the particle energy flux and (2) the particle energy stored in the square domain $S_{d}$. Figure 5 plots the temporal evolution of four components of the particle energy integrated over the square domain $S_{d}$ for Cases B (left) and D (right), where the solid, dashed, dot-dashed, and dotted lines stand for the ion thermal energy $W_{T, i o n}$, the electron thermal energy $W_{T, \text { ele }}$, the ion kinetic energy $W_{K, \text { ion }}$, and the electron kinetic energy $W_{K, e l e}$, respectively. The particle energies for the multi-island case (Case D) are about twice as large as those for the no-island case (Case B). The ion thermal energy (solid line) is the largest among the four energies for the both cases, and the electron kinetic energy (dotted line) is the smallest. Consequently, the total ion energy is about twice as large as the total electron energy. In this way, the central current sheet with magnetic islands can store larger particle energy, as compared with the current sheet without any magnetic island. 

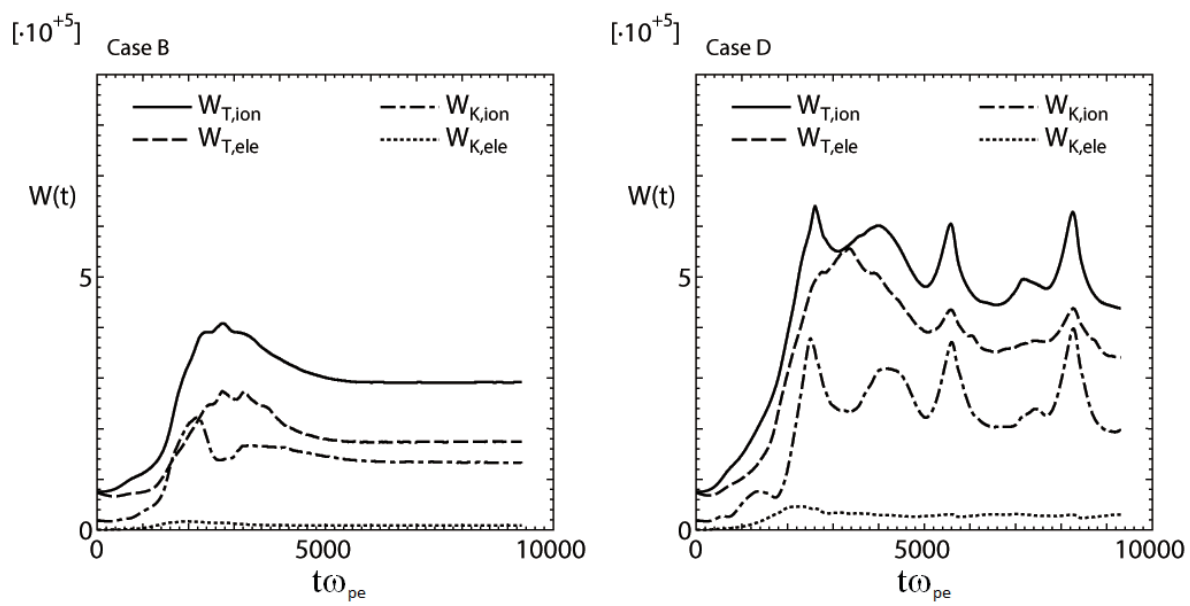

Figure 5. Temporal evolution of the particle energy integrated over the simulation domain for Cases B (left) and D (right) where the solid, dashed, dot-dashed, and dotted lines stand for the ion thermal energy, the electron thermal energy, the ion kinetic energy, and the electron kinetic energy, respectively.

Figure 6 shows the spatial profiles of the electron (left) and ion (right) temperatures at three different time periods for Case $\mathrm{D}$, where the particle temperature is defined by the average of the diagonal components of the pressure tensor divided by $n_{i / e} M_{e} c^{2}$ with the ion or electron number density $n_{i / e}$. Here, the periods $\omega_{p e} t=5168$ and $\omega_{p e} t=7753$ correspond to the times when the magnetic island grows to its maximum size, and the intermediate period $\omega_{p e} t=6461$ corresponds to the time when the first large magnetic island moves out of the simulation domain.
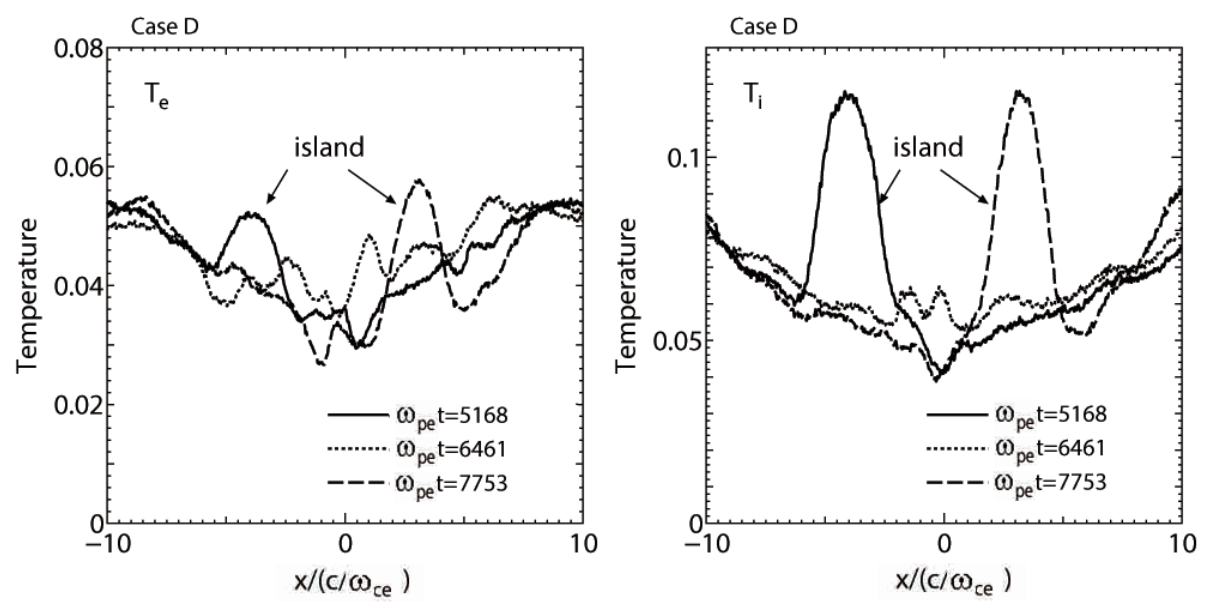

Figure 6. Spatial profiles of the electron (left) and ion (right) temperatures at three different time periods for Case D, where solid, dotted, and dashed curves stand for the profiles at $\omega_{\text {pe }} t=5168$, $\omega_{p e} t=6461$, and $\omega_{p e} t=7753$, respectively.

Both profiles are peaked at the center of magnetic islands at the periods $\omega_{p e} t=5168$ and $\omega_{p e} t=7753$, while there is no such a peaked profile at the no-island state of $\omega_{p e} t=6461$. The peaked value of the ion temperature is about twice as large as that of the electron temperature. These results suggest that a magnetic island plays an important role as a particle energy container which can store a large amount of the particles with the high temperature, and carry them from the reconnection region.

Next, let us consider how the particles (specifically, the ions) are heated in the presence of the magnetic islands. The dominant mechanisms leading to the ion heating are the ion viscous heating and the ion compressional heating, the rates of which are approximately given by the two-fluid formulations as $H_{\text {visc }}=-\pi_{i, j} \partial_{i} u_{j}$ and $H_{c o m p}=-5 / 2 p(\nabla \cdot \mathbf{u})$, where the stress tensor is assumed to 
be expanded into scalar and off-diagonal components as $\sigma_{i, j}=-p \mathbf{I}_{i, j}-\pi_{i, j}$. Figure 7 shows the spatial profiles of the ion viscous heating rate (top) and ion compressional heating rate (bottom) at the island growing phase of $\omega_{p e} t=7494$ for Case D.

It is clearly seen in Figure 7 that the ion compressional heating rate becomes dominant inside the magnetic island, while there is no clear structure in the ion viscous heating rate. This phenomenon can be explained as follows. For the no-island case, most of the ions are accelerated by the electrostatic field when they pass across the magnetic separatrix, and move into the downstream region. For the one-island case, however, two reconnection points exist on the both side of a magnetic island. A left wing of the magnetic separatrix corresponding to the right reconnection point is connected with the field line covering the magnetic island, and a right wing of the magnetic separatrix corresponding to the left reconnection point is also connected with the field line. In other words, the magnetic separatrix lines starting from the two reconnection points wrap the magnetic island located inbetween. Thus, the high-energy ion flows moving into the magnetic island are supplied from the upstream regions of the two reconnection points. The convergent ion flows are trapped and are mixed inside the magnetic island in the presence of a strong magnetic field, as shown in Figure 8. In this way, the compressional ion heating mechanism is more effective for the multi-island case compared with the no-island case.

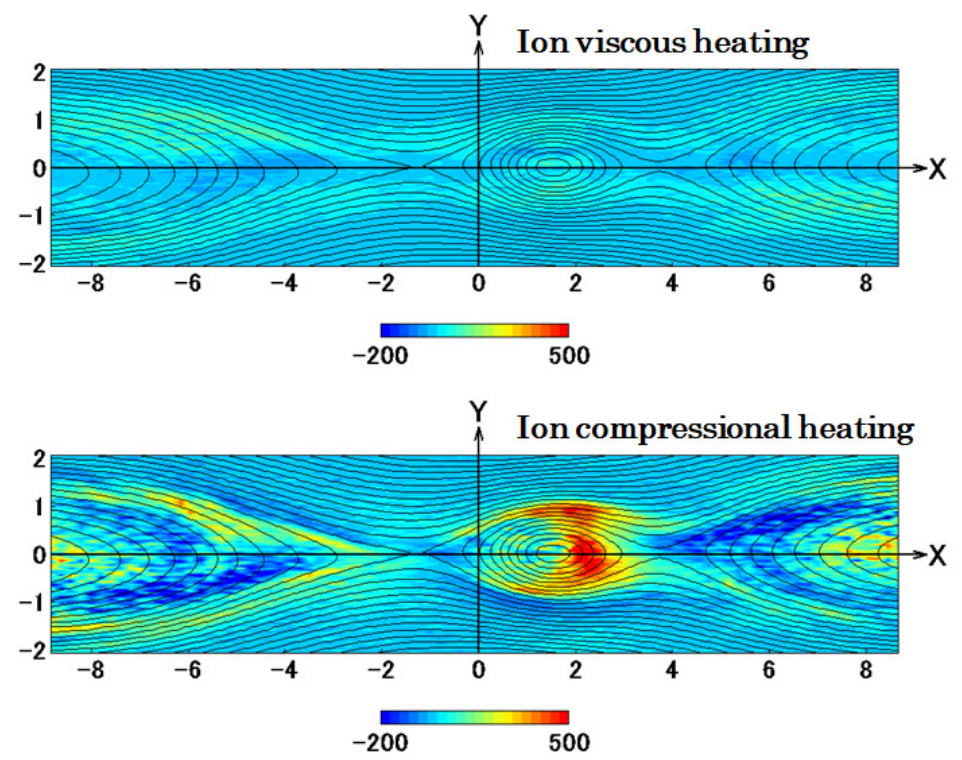

Figure 7. Spatial profiles of the ion heating rate (color contours) and the magnetic field lines (black lines) at the island growing phase of $\omega_{p e} t=7494$ for Case D, where the top and bottom panels stand for the ion viscous heating rate and ion compressional heating rate, respectively.

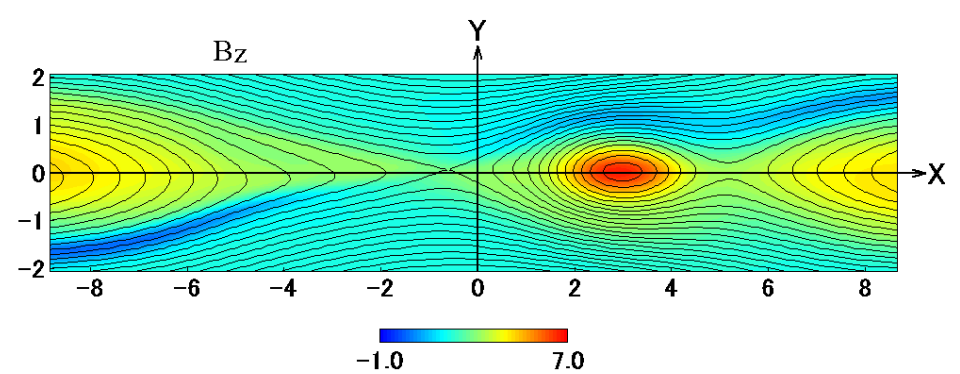

Figure 8. Spatial profile of the $z$-component of the magnetic field $B_{z}$ at $\omega_{p e} t=7753$ for Case D. 


\section{Discussion}

We have investigated the role of magnetic islands in collisionless driven reconnection from the standpoint of a kinetic approach to multi-scale phenomena by means of two-dimensional PIC simulation. When the ratio of the inflow window size to the current sheet width $X_{\text {slot }} /\left(2 l_{m i}\right)$ becomes larger than the critical value $R_{c r}\left(6.166<R_{c r}<8.720\right)$, an intermittent behavior accompanied by the multi-island formation appears in the dynamical evolution of a reconnection system. This suggests that this multi-island formation process can be explained in relation to the excitation of a tearing instability $[6,18,23]$. The electromagnetic energy is more effectively transferred to the particle energy in the multi-island case compared with that in the no-island case. The transferred energy is stored inside the magnetic island in the form of the thermal energy through the compressional heating, and is carried away together with the magnetic island from the reconnection region. This result suggests that a chain of magnetic islands may have the potential to widen the dissipation region on a global scale, and to strengthen the energy dissipation rate, as indicated by many papers [6,11-17,24].

However, several problems remain unresolved in relation to the role of magnetic islands in the multi-scale magnetic reconnection phenomena. Firstly, the following unanswered questions remain: Which physics determine the time scales so that a magnetic island can grow and move out from the simulation domain in an externally driven system? Is the formation of magnetic islands intermittent or periodic? In order to answer these questions, we need much longer time scale simulation runs.

The second problem relates to the dynamics of a multi-island system on a global scale. This paper deals with the formation and dynamics of only a few magnetic islands. In general, however, many magnetic islands with different spatial sizes are generated and evolve dynamically through plasma instabilities, merging, turbulence, and other processes. Particle acceleration and heating also take place in the evolving multi-island system through the Fermi acceleration [14,24].

The third problem is with respect to influence of the boundary model. In the PASMO code, an external driving field, which takes a uniform and constant profile after an initial period, is assumed at the upstream boundary in order to supply plasmas into a microscopic reconnection system from a surrounding macroscopic system. In general, the microscopic reconnection system is always under the influence of the macroscopic system which evolves dynamically with time. Therefore, the model of the external driving field should be extended to more realistic one so as to include their mutual interaction. It would thus be very interesting to clarify how the microscopic multi-island state is modified when the external driving field is oscillating on a macroscopic time scale.

The fourth problem relates to how to interlock the microscopic system with the macroscopic system self-consistently. Because the PASMO code has been developed for a kinetic system which is open to the surrounding macroscopic system, the system size is too small to deal with hierarchically connecting chains of magnetic islands. In order to solve this problem, we need a multi-hierarchy simulation code which can deal with both microscopic physics and macroscopic physics, consistently and simultaneously. The MHD-PIC interlocked model is a powerful tool for this [25]. We will apply this model to the formation of a chain of magnetic islands on a global scale.

Acknowledgments: The simulation was performed by employing the plasma simulator at the National Institute for Fusion Science (NIFS). This study was partially supported by general coordinated research at the NIFS (NIFS17KNSS097, NIFS17KNXN357).

Conflicts of Interest: The founding sponsors had no role in the design of the study; in the collection, analyses, or interpretation of data; in the writing of the manuscript, and in the decision to publish the results.

\section{References}

1. Yamada, M. Progress in understanding magnetic reconnection in laboratory and space astrophysical plasmas. Phys. Plasmas 2007, 14, 058102. [CrossRef]

2. Yamada, M.; Kulsrud, R.; Ji, H. Magnetic Reconnection. Rev. Mod. Phys. 2010, 82, 603-664. [CrossRef]

3. Horiuchi, R.; Sato, T. Collisionless driven reconnection in an open system. Earth Planets Space 2001, 53, 439-445. [CrossRef] 
4. Pei, W.; Horiuchi, R.; Sato, T. Long time scale evolution of collisionless driven reconnection in a two-dimensional open system. Phys. Plasmas 2001, 8, 3251-3257. [CrossRef]

5. Fujimoto, K.; Sydora, R. Plasmoid-Induced Turbulence in Collisionless Magnetic Reconnection. Phys. Rev. Lett. 2012, 109, 265004. [CrossRef]

6. Loureiro, N.F.; Uzdensky, D.A. Magnetic reconnection: From the Sweet-Parker model to stochastic plasmoid chains. Plasma Phys. Control. Fusion 2016, 58, 014021. [CrossRef]

7. Tuneta, S.; Hara, H.; Shimizu, T.; Acton, L.W.; Strong, K.T.; Hudson, H.S.; Ogawara, Y. Observation of a solar flare at the limb with the YOHKOH soft X-ray telescope. Publ. Astron. Soc. Jpn. 1992, 44, L63-L69.

8. Sweet, P.A. The Neutral Point Theory of Solar Flares. In Electromagnetic Phenomena in Cosmical Physics; Lehnert, B., Ed.; Cambridge U.P.: New York, NY, USA, 1958; p. 123.

9. Parker, E.N. Sweet's Mechanism for Merging Magnetic Fields in Conducting Fluids. J. Geophys. Res. 1957, 62, 509-520. [CrossRef]

10. Horiuchi, R.; Den, M.; Tanaka, T.; Ohatni, H.; Usami1, S. Macro- and microphysics of magnetic reconnection in a multi-hierarchy open system. Plasma Phys. Control. Fusion 2013, 55, 014008. Available online: http:/ /iopscience.iop.org/0741-3335/55/1/014008 (accessed on 19 April 2018). [CrossRef]

11. Shibata, K.; Tanuma, S. Plasmoid-induced-reconnection and fractal reconnection. Earth Planets Space 2001, 53, 473-482. [CrossRef]

12. Phan, T.D.; Gosling, J.T.; Davis, M.S.; Skoug, R.M.; Øieroset, M.; Lin, R.P.; Lepping, R.P.; McComas, D.J.; Smith, C.W.; Reme, H.; et al. A magnetic reconnection X-line extending more than 390 Earth radii in the solar wind. Nature 2006, 439, 175-178. [CrossRef]

13. Scime, E.; Cekic, M.; Den Hartog, D.J.; Hokin, S.; Holly, D.J.; Watts, C. Ion heating and magnetohydrodynamic dynamo fluctuations in the reversed-field pinch. Phys. Rev. Lett. 1992, 68, 2165-2168. [CrossRef]

14. Drake, J.F.; Shay, M.A.; Thongthai, W.; Swisdak, M. Production of Energetic Electrons during Magnetic Reconnection. Phys. Rev. Lett. 2005, 94, 095001. [CrossRef]

15. Eriksson, S.; Lapenta, G.; Newman, D.L.; Phan, T.D.; Gosling, J.T.; Lavraud, B.; Khotyaintsev, Y.V.; Carr, C.M.; Markidis, S.; Goldman, M.V. On Multiple Reconnection X-Lines and Tripolar Perturbations of Strong Guide Magnetic Fields. Astrophys. J. 2015, 805, 43,-637X/805/1/43. [CrossRef]

16. Wang, H.; Lu, Q.; Huang, C.; Wang, S. Electron acceleration in a secondary magnetic island formed during magnetic reconnection with a guide field. Phys. Plasmas 2017, 24, 052113. [CrossRef]

17. Matthaeus, W.H.; Ambrosiano, J.J.; Goldstein, M.L. Particle Acceleration by Turbulent Magnetohydrodynamic Reconnection. Phys. Rev. Lett. 1984, 53, 1449. [CrossRef]

18. Markidis, S.; Henri, P.; Lapenta, G.; Divin, A.; Goldman, M.; Newman, D.; Laure, E. Kinetic simulations of plasmoid chain dynamics. Phys. Plasmas 2013, 20, 082105. [CrossRef]

19. Ohtani, H.; Horiuchi, R. Open boundary condition for particle simulation in magnetic reconnection research. Plasma Fusion Res. 2009, 4, 024. [CrossRef]

20. Birdsall, C.K.; Langdon, A.B. Plasma Physics via Computer Simulation; McGraw-Hill: New York, NY, USA, 1985; p. 351.

21. Harris, E. On a plasma sheath separating regions of oppositely directed magnetic field. Nuovo Cimento 1962, 23, 115-121. [CrossRef]

22. Horiuchi, R.; Sato, T. Particle Simulation Study of Collisionless Driven Reconnection in a Sheared Magnetic Field. Phys. Plasmas 1997, 4, 277-289. [CrossRef]

23. Furth, H.P.; Killeen, J. Finite-Resistivity Instabilities of a Sheet Pinch. Phys. Fluids 1963, 6, 459-484. [CrossRef]

24. Hoshino, M. Stochastic Particle Acceleration in Multiple Magnetic Islands during Reconnection. Phys. Rev. Lett. 2012, 108, 135003. [CrossRef]

25. Usami, S.; Horiuchi, R.; Ohtani, H.; Den, M. Development of multi-hierarchy simulation model with non-uniform space grids for collisionless driven reconnection. Phys. Plasmas 2013, 20, 061208. [CrossRef]

(C) 2018 by the authors. Licensee MDPI, Basel, Switzerland. This article is an open access article distributed under the terms and conditions of the Creative Commons Attribution (CC BY) license (http:/ / creativecommons.org/licenses/by/4.0/). 